\section{Hierro intravenoso en la terapia del síndrome de piernas inquietas}

\author{
MARCELO MIRANDA C. ${ }^{1}$, M. LEONOR BUSTAMANTE C. ${ }^{2}$
}

\section{Intravenous iron for restless legs syndrome. Report of five cases}

Restless legs syndrome (RLS) may severely affect the quality of life of patients. A deficient iron incorporation into the central nervous system has an important role in the pathophysiology of RLS. Severely affected patients may not respond to current therapeutic options. We report a preliminary experience with five patients with severe RLS and low serum ferritin levels who did not improve with oral iron. All were treated with $1 \mathrm{~g}$ of intravenous iron carboxymaltose. They experienced a marked improvement in symptoms, evident even during the first week of therapy that had persistent after up to two years of follow-up. A significant change in the RLS severity scale was observed after intravenous iron. Serum ferritin levels increased in all of them. Intravenous iron could be a therapeutic option for patients with severe RLS.

(Rev Med Chile 2019; 147: 1569-1571)

Key words: Iron: Restless Legs Syndrome; Therapeutics.
'Departamento Neurología

Clínica Las Condes y Fundación Diagnosis. Santiago, Chile. 2Programa de Genética Humana, Instituto de Ciencias Biomédicas y Departamento de Psiquiatría y Salud Mental Norte, Facultad de Medicina, Universidad de Chile.

Santiago, Chile.

Trabajo no recibió financiamiento. Los autores declaran no tener conflictos de interés.

Recibido el 1 de julio de 2019, aceptado el 26 de noviembre de 2019.

Correspondencia a:

Dr. Marcelo Miranda Departamento Neurología Clínica Las Condes. Santiago, Chile. marcelomirandac@gmail.com
$\mathrm{E}$ 1 síndrome de piernas inquietas (SPI) consiste en la imperiosa necesidad de mover las piernas al estar en reposo e intentar dormir y puede llegar a afectar severamente la calidad de vida de los afectados ${ }^{1-3}$. Es altamente prevalente en medicina alcanzando $10 \%$ de la población gene$\mathrm{ral}^{1}$. La terapia habitual consiste en uso de agentes dopaminérgicos y de preferencia de fármacos como pregabalina o gabapentina ${ }^{4-6}$.

En la fisiopatología del SPI es básico una deficiente incorporación de hierro al sistema nervioso central y a las neuronas que sintetizan dopamina $(7,9)$. Un indicador del trastorno de la incorporación de hierro es la presencia de bajos niveles de ferritina. Se acepta que los valores óptimos de ferritina para considerar o no terapia con hierro deben ser sobre $70 \mathrm{mcg} / \mathrm{l}^{9}$; bajo ese valor se justifica hacer una terapia con hierro. La terapia con hierro oral, generalmente, no es bien tolerada y es lenta en lograr subir ferritina por dificultades en su absorción'.

Por ello en los últimos años ha surgido la opción de tratar a los pacientes con síntomas más severos de SPI con hierro intravenoso y con muy buenos resultados?.

Se desea comunicar una experiencia preliminar con esta terapia en los últimos 2 años en que hemos tratado pacientes con SPI refractario a la terapia habitual.

\section{Pacientes y Método}

Los pacientes corresponden a cinco enfermos (1 hombre) (rango edad: 65-80 años), sólo 1 de ellos con anemia evidente en hemograma, y quienes presentaban un severo síndrome de piernas inquietas con insuficiente respuesta a la terapia habitual y en quienes los niveles de ferritina estaban bajos de lo recomendado $(<70 \mathrm{mcg} / \mathrm{L})$, rango detectado: 4-20). Si bien el rango normal de ferritina es $>12 \mathrm{mcg} / \mathrm{L}$ para un laboratorio standard (es el límite aceptado para la anemia ferropriva), el Grupo Internacional de Estudio de SPI ha determinado que $>70 \mathrm{mcg} / \mathrm{L}$ es lo óptimo para estos pacientes y que el objetivo de la terapia con hierro es lograr ese valor $(6,9)$. 
En cuatro de los cinco pacientes, la causa de la deficiencia de hierro se atribuyó a origen ginecológico; en el hombre se debió a hemorragia digestiva.

Previo consentimiento escrito, se administró una inyección intravenosa de $1 \mathrm{~g}$ de hierro carboximaltosa i.v en forma ambulatoria por 1 vez. El protocolo fue aprobado por el Comité de Ética local.

Los síntomas fueron medidos previo a la inyección y luego de un período de al menos seis semanas de administrada la dosis. La severidad fue medida por la escala de 10 ítems propuesta por Walters $\mathrm{y} \mathrm{col}^{1}$, que incluye 10 parámetros como intensidad de síntomas, necesidad de moverse, alivio obtenido, alteración del sueño, cansancio derivado de estas molestias, frecuencia semanal, duración diaria, interferencia con actividades diarias y consecuencias en el ánimo. Cada uno de estos ítems se cuantifica de 0 (nunca) a 4 (muy severo). Se clasifica así la severidad de SPI de acuerdo al puntaje: leve (0-10), moderado (11-20), grave (21-30), muy grave (31-40).

\section{Análisis estadístico}

Considerando que la terapia con hierro en SPI se ha introducido recientemente en nuestro medio, el presente trabajo reporta la experiencia con una muestra pequeña de pacientes. Previamente, se ha establecido que con muestras $<=5$ es posible obtener conclusiones a partir de la comparación de medias con una probabilidad de error tipo I de $5 \%$ (nivel de significancia 0,05 ) y con una potencia adecuada ( $>80 \%)$ sólo en los casos en los que el tamaño del efecto del tratamiento es grande (es decir, d de Cohen $>2)^{10}$. Un metaanálisis de estudios estableció una diferencia significativa entre el uso de hierro y placebo con un d de Cohen de $-3,78^{11}$. Con estos antecedentes, de acuerdo a la recomendación de Winter ${ }^{10}$, usamos la prueba $\mathrm{t}$ de student de una cola para comparar la media en el puntaje pre y postintervención, por medio del calculador Vassarstats (disponible en http:// vassarstats.net/index.html).

\section{Resultados}

Todos los pacientes experimentaron una respuesta satisfactoria. No se observaron efectos colaterales serios; 2 pacientes comunicaron cefalea de leve magnitud durante la infusión. El puntaje promedio en el grupo preintervención fue 37,4 (rango 30-40), es decir, todos los pacientes tenían una enfermedad grave o muy grave. Postintervención, el promedio fue 8 (rango 3-12), quedando uno de los pacientes en la categoría de enfermedad moderada y el resto leve. La comparación de las medias entre los dos grupos fue significativamente diferente $(\mathrm{p}=0,0003275)$. Al momento de la reevaluación, todos los pacientes presentaban niveles aceptables de ferritina (mayor de $70 \mathrm{mcg} / \mathrm{l})$.

\section{Discusión}

El síndrome de piernas inquietas es uno de los trastornos más frecuentes en medicina y, a menudo, es subdiagnosticado y mal tratado ${ }^{2,4}$.

Por el impacto que tiene esta condición en la vida del paciente debe ser adecuadamente diagnosticada y tratada por todo médico no sólo el especialista ${ }^{12-14}$.

En la última década los avances en el estudio de su fisiopatología ponen como factor básico una deficiente incorporación de hierro a nivel de neuronas dopaminérgicas lo que lleva secundariamente a defectos en la neurotransmisión dopaminérgica, pero también en vías glutamatérgicas y otras $^{7-9}$. Los tratamientos actuales sólo van a tratar los efectos que producen la depleción de hierro y no el problema de fondo.

Los fármacos para el tratamiento de SPI no están exentos de reacciones adversas como es en el caso de los dopaminérgicos la aparición de un síndrome de descontrol de impulsos y agravación de los síntomas con la terapia a largo plazo (fenómeno de potenciación $)^{15-17}$.

La corrección de bajos niveles de hierro (expresados en una baja ferritina) por vía i.v constituye una buena opción para casos severos del SPI e incluso se ha sugerido que debe ser considerada una de las terapias de primera línea especialmente aquellos pacientes con niveles de ferritina menor de $100 \mathrm{mg} / \mathrm{l}^{9}$. Si bien esta comunicación tiene la limitación de un número bastante reducido de pacientes, apoya la evidencia actual cada vez más sólida de que esta modalidad de terapia es útil en SPI, especialmente, en pacientes que no logran mejoría con aporte de hierro oral o quienes no lo toleran. Uno de los cinco pacientes logró suspender toda otra 
terapia para SPI y los otros cuatro la redujeron considerablemente a más de la mitad.

En cuanto a evidencia, si bien una revisión Cochrane del año 2012 no encontraba mayor utilidad del uso de hierro en $\mathrm{SPI}^{18}$, una nueva revisión más exhaustiva del presente año sí apoya su uso ${ }^{11}$.

Las nuevas formulaciones de hierro intravenoso como la utilizada en esta experiencia no conllevan el riesgo de anafilaxia que era el riesgo mayor de las formulaciones más antiguas, y son así consideradas seguras en su uso9. Los síntomas más frecuentes asociados al uso de hierro intravenoso son náuseas y cefalea, ambos de leve intensidad. Sólo dos pacientes comunicaron algún efecto secundario y éste fue cefalea y de leve intensidad y transitoria. Se recomienda que la infusión se lleve a cabo en forma lenta en un período de 1 hora y bajo supervisión de enfermera en una unidad de terapia ambulatoria ${ }^{9}$. Un inconveniente de esta terapia es el costo: es de alto costo, pero considerando la potencial reducción de la terapia oral usada por el paciente y el beneficio en calidad de vida, la relación costo beneficio puede ser favorable.

Por el impacto que tiene esta condición en la vida del paciente creemos importante considerar esta terapia como una opción de tratamiento no sólo sintomático sino eventualmente curativo al influir directamente en el mecanismo fisiopatológico de SPI.

\section{Referencias}

1. Allen RP, Picchietti D, Hening WA, Trenkwalder C, Walters AS, Montplaisir. Restless legs syndrome: diagnostic criteria, Sleep Med 2003; 4: 101-19.

2. Miranda M, Araya F, Castillo JL, Durán C, González F, Arís L. Síndrome de piernas inquietas: Estudio clínico en población general adulta y en pacientes urémicos. Rev Med Chile 2001; 129: 179-86.

3. Szentkiralyi A, Fendrich K, Hoffmann W. Incidence of restless legs syndrome in two population-based cohort studies in Germany. Sleep Med 2011; 12: 815-20.

4. Miranda M, Hudson L. Síndrome de piernas inquietas (enfermedad de Willis-Ekbom): seguimiento a largo plazo de una serie de pacientes. Rev Med Chile 2016; 4 (12): 1561-6.

5. Earley CJ. Clinical practice. Restless legs syndrome. N Engl J Med 2003; 348: 2103e9.

6. García-Borreguero D, Kohnen R, Silber MH. The long- term treatment of restless legs syndrome/Willis-Ekbom disease: evidence-based guidelines and clinical consensus best practice guidance: a report from the International Restless Legs Syndrome Study Group. Sleep Med 2013; 14: 675-84.

7. Allen R. Dopamine and iron in the pathophysiology of restless legs syndrome (RLS). Sleep Med 2004; 5: 385-91.

8. Allen RP, Earley CJ. The role of iron in restless legs syndrome. Mov Disord 2007; 22: S440e8

9. Allen R, Picchietti D, Auerbach M, Cho YW, Connor J, Earley Ch, García-Borreguero D. On behalf of the International Restless Legs Syndrome Study Group (IRLSSG). Evidence-based and consensus clinical practice guidelines for the iron treatment of restless legs syndrome/Willis-Ekbom disease in adults and children: an IRLSSG task force report Sleep Medicine 2018; 41: 27-44.

10. De Winter JCF. Using the Student's t-test with extremely small sample sizes. Practical Asessment Research and evaluation. 2013; 18: 1-10.

11. Trotti LM, Becker LA. Iron for the treatment of restless legs syndrome. Cochrane Database Syst Rev 2019; 1: CD007834. doi:10.1002/14651858.CD007834.pub3.

12. Earley CJ, Silber MH. Restless legs syndrome: understanding its consequences and the need for better treatment. Sleep Med 2007; 11: 807e15.

13. Mitterling T, Heidbreder A, Stefani A, Fritz J, Ulmer H, Poewe W. Natural course of restless legs syndrome/ Willis-Ekbom disease: long-term observation of a large clinical cohort. Sleep Med 2015; 1252-8.

14. Fuhs A, Bentama D, Antkowiak R. Effects of short-and long-term variations in RLS severity on perceived health status-the COR Study. PLoS ONE 2014; 9 (4): e9482 doi: 10.1371/journal.pone.0094821.

15. García-Borreguero D, Williams AM. Dopaminergic augmentation of restless legs syndrome. Sleep Med Rev 2010; 14: 339-46.

16. García-Borreguero D, Allen RP, Kohnen R, Hogl B, Trenkwalder C, Oertel W. Diagnostic standards for dopaminergic augmentation of restless legs syndrome: report from a World Association of Sleep Medicine International Restless Legs Syndrome Study Group consensus conference at the Max Planck Institute. Sleep Med 2007; 8: 520-3.

17. Cornelius JR, Tippmann-Peikert M, Slocumb NL, Frerichs $\mathrm{CF}$, Silber $\mathrm{MH}$. Impulse control disorders with the use of dopaminergic agents in restless legs syndrome: a case-control study. Sleep 2010; 33: 81-7.

18. Trotti LM, Bhadriraju S, Becker LA. Iron for restless legs síndrome. Cochrane Database Syst Rev 2012; (5): CD007834. doi: 10.1002/14651858.CD007834.pub2. 